\title{
Research on Dynamic Updating of Grid Service
}

\author{
Jiankun Wu, Linpeng Huang, and Dejun Wang \\ Department of Computer Science, Shanghai Jiaotong University, Shanghai, 200240, \\ P.R. China \\ jkwu@sjtu.edu.cn, huang-lp@cs.sjtu.edu.cn, wangdejun@sjtu.edu.cn
}

\begin{abstract}
In complicated distributed system based on grid environment, the grid service is inadequate in the ability of runtime updating. While in the maintenance of systems in grid environment, it is an urgent issue to solve to support the transparent runtime updating of the services, especially in the case of services communicating with each other frequently. On the basis of researches on the implementation of grid services and interaction between them following WSRF [3], this paper introduces proxy service as the bridge of the interaction between services and achieved the ability to support the runtime dynamic updating of grid services. Gird service updating must happen gradually, and there may be long periods of time when different nodes run different service versions and need to communicate using incompatible protocols. We present a methodology and infrastructure that make it possible to upgrade grid-based systems automatically while limiting service disruption.
\end{abstract}

Keywords: Grid service, Dynamic updating, Proxy service, Simulation service.

\section{Introduction}

With the change of application requirements and wide use of Internet, the across-area and across-organization complicated applications have developed greatly in various fields. The distributed technology has become the main method in these applications. Accompanying with the system expanding day by day, the maintenance and modification of the system became more frequent. Research shows that nearly half of costs are spent in the maintenance of the complicated distributed system. Services have to be paused in the traditional procedure of software maintenance, but some system such as Bank must provide services continuously in 24 hours, any short-time pause will make great lost. How to resolve the dilemma? Answer is Dynamic Updating technology. Software Updating is defined as the dynamic behavior including software maintenance and update in the life-cycle of software system [6]. Due to maintaining the system with whole system working normally, Dynamic Updating is significant. The grid computing technology is the latest achievement of the development of distributed technology, aiming to resolve the resource share and coordination in WAN distributed environment and avoid the drawbacks such as inadequate computation ability or unbalance loads[1][7][8]. It is a trend to develop new complicated system based on grid technology and transplant the current system into grid environment. 
As application system based on grid technology consists of services with specific function, the system maintenance is mainly maintaining services. As the same as other distributed systems, the services maintenance in grid application system still face the problem of terminating service. Therefore it is necessary to introduce Dynamic Updating technology in the service maintenance of grid environment. It has more practical significance especially when the built system is in its long-time running.

In the current grid technology, if we want to update or modify the working grid services, we must stopped and start new service to run. This model is inadequate in dynamic switch in running service. The substitution of grid service will make part of the system terminated or produce long delay, especially in the case of service communicate with each other frequently.

The proxy service and simulation service are introduced in the architecture supporting grid service updating. The proxy service is not only responsible for the service requests transmitting but also responsible for contacting with updating component information service through subscribe/publish styles to obtain the new version information in time. The interaction and interface of different service will be transparency by introducing proxy service. And the simulation service is responsible for simulating of behavior and state format between different versions of service.

This paper presents a flexible and efficient updating method that enables gridbased systems to provide service during updating. We present a new methodology that makes it possible to updating grid-based systems while minimizing disruption and without requiring all upgrades to be compatible. The rest of paper is organized as follow. In section 2, the updating requirements of grid service is discussed. In section 3, the architecture and relative technology supporting grid service updating are presented and discussed. In section 4, prototype system and relative tests are described. Finally, summary and future works are given.

\section{Architecture Supporting Grid Service Updating}

The architecture models a grid-based system as a collection of grid services. A service has an identity, a collection of method that defines its behavior, and a collection of resource representing state. Services communicate by sending soap message. A portion of a service's state may be persistent. A node may fail at any point; when it node recovers, the service reinitializes itself from the persistent portion of its state and when updating, the persistent state may need change the data format for the new version.

To simplify the presentation, we assume each node runs a single top-level service that responds to remote requests. Thus, each node runs a top-level service-proxy grid service. An upgrade moves a system from one version to the next by specifying a set of service updating, one for each grid service that is being replaced. The initial version has version number one and each subsequent version has the succeeding version number. 


\subsection{System Architecture}

A class updating has six components: <oldService, newService, TF, SF, pastSimulationService, futureSimulationService>. OldService identifies the service that is now obsolete; newService identifies the service that is to replace it. TF identifies a transform function that generates an initial persistent state for the new service from the persistent state of the old one. SF identifies a scheduling function that tells a node when it should update. PastSimulationService and futureSimulationService identify services for simulation objects that enable nodes to interoperate across versions. A futureSimulationService allows a node to support the new service's behavior before it upgrades; a pastSimulationService allows a node to support the old service's behavior after it upgrades. These components can be omitted when not needed.

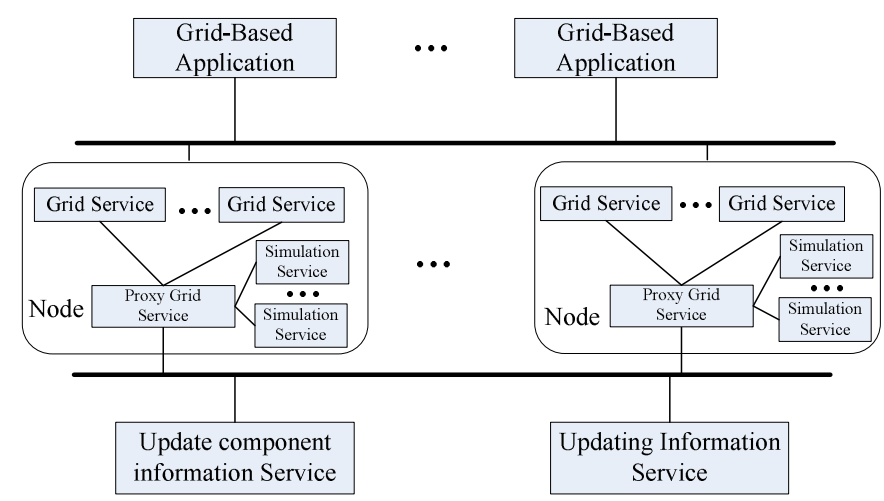

Fig. 1. Updating architecture

\subsection{Analysis of Proxy Service Mechanism}

The object of proxy service introducing is obtaining the transparency between services. When the grid service is updating, the other grid services in the same grid system will be not aware of it. The proxy service is not only responsible for the service requests transmitting but also responsible for contacting with updating component information service through subscribe/publish styles to obtain the new version information in time.

\subsection{Version Management}

Because the updating doesn't complete in twinkling, it is necessary to support the multi version coexist at same time. The simulation service is responsible for the simulating of different interface and saving and recovering of states between current version and old version, current version and new version services. 
In order to accurately locate the simulation service of relative version for proxy service, each simulation service has a resource to hold the version information such as request interfaces, parameter formats, URL for the software data and so on.

When the service updating happens, the work flow of the proxy service is showed as figure 3. The Proxy service gets the simulation service according to relative resource and then deliver the service request to the respect simulation service and return the result to the service requester in the finally.

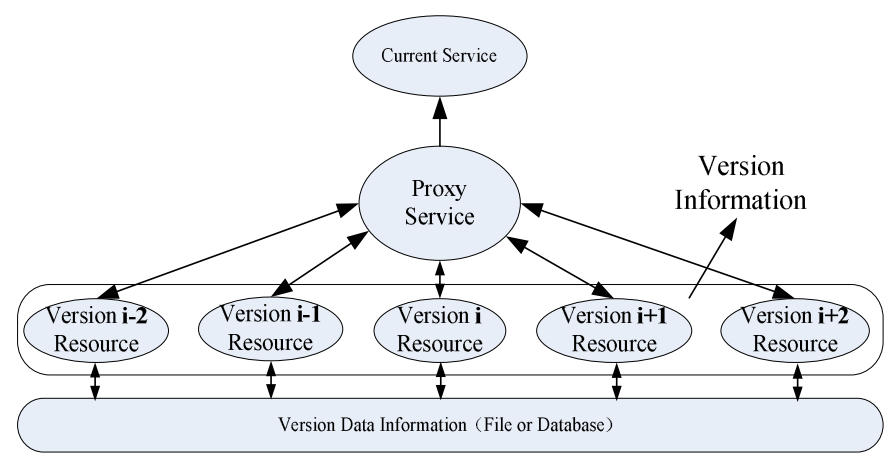

Fig. 2. Version information in service updating

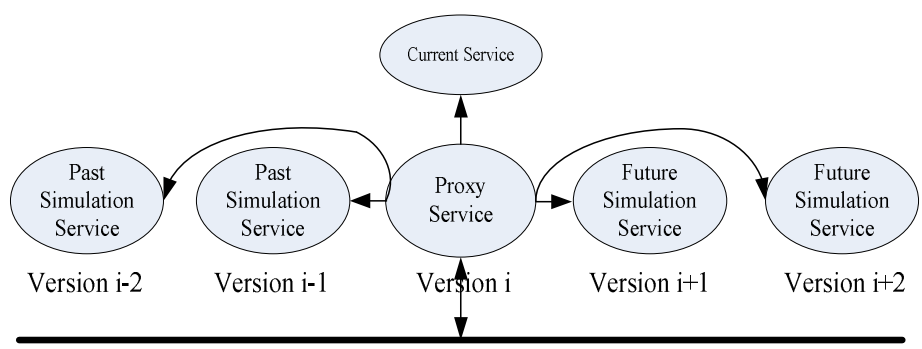

Fig. 3. Simulation procedure

\subsection{Subscribe/Publish Model in Updating Procedure}

The subscribe/publish style is adopted for publishing service version changing information in order to make the updating information report more quickly and reduce the load of network. The proxy service in every grid node subscribes to the updating component information service for the services version changing information. Due to the proxy service receiving the information, it will make some actions according to the relationship between current service in the node and the new service. This style is more efficient than the continue request/report method in the traditional updating system. It makes the nodes deployed grid service focus on the main computing task without initiatively querying the new service version information all the time.

As showed in figure 4, the proxy service is activated as a basic service when the grid service container starts running. At the same time, the proxy service initiatively 
subscribe to the service version changing information of updating component information service. The new version grid service is stored in the grid data base and the URL of the data base is hold in the resource of updating component information service. As the proxy services aware of the new service information of current service, it requests the Grid Ftp with URL information to transmit the software data to current node and deploys it. It reports the updating mid-states through request/reports method to updating information service and the states are also represented by resource which hold the URL of the data base storing the mid-states.

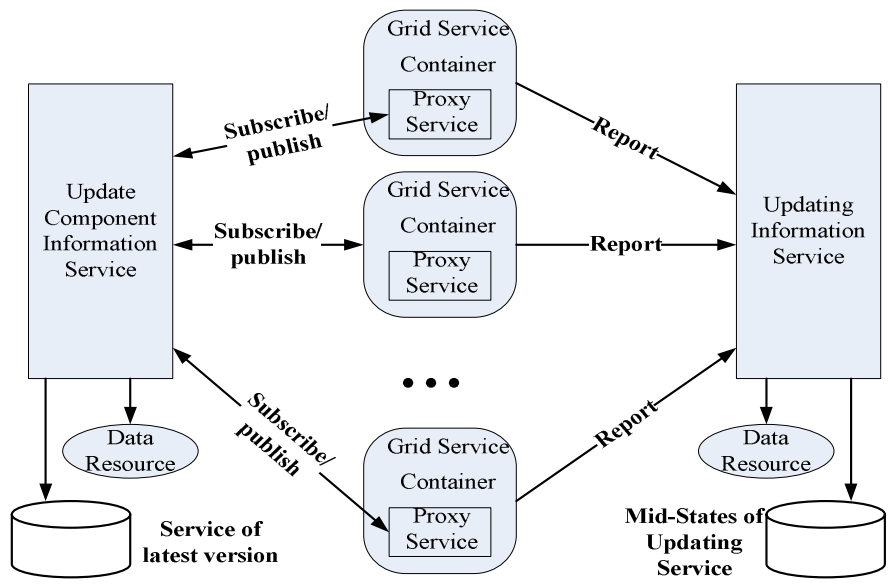

Fig. 4. Interaction model in updating platform

\subsection{Scheduling of Service Management}

We could add filters to the model that would determine some subset of nodes that need to upgrade. Adding filters is enough to allow restructuring a system in arbitrary ways.

In order to make the grid service dynamic updating more efficient, dynamic grid service updating scheduling which is based on monitoring the load of nodes is adopted in this paper. The performance evaluating model that bases on CPU frequency, CPU load, memory capacity, occupied proportion of memory, disk capacity and occupied proportion of disk is built. This model could make the updating procedure more efficient and reduce the service interruption. We adopt the following formula to define the evaluation.

$$
\begin{gathered}
\omega=\omega_{C P U}+\omega_{M E M}+\omega_{D I S K} \\
\omega_{C P U}=p_{C P U_{-} F r e q} * C P U_{-} F r e q+p_{C P U_{-} \text {Load }} * C P U_{-} \text {Load } \\
\omega_{M E M}=p_{M E M_{-} \text {Cap }} * M E M M_{-} \text {Cap }+p_{M E M_{\text {_occupied }}} * M E M_{-} \text {Occupied }
\end{gathered}
$$




$$
\omega_{\text {DISK }}=p_{\text {DISK_Cap }} * D I S K_{-} C a p+p_{\text {DISK_occupied }} * D I S K_{-} \text {Occupied }
$$

In the above formula, $\omega$ is the final evaluating parameter, $\omega_{C P U}$ is $\mathrm{CPU}$ evaluating parameter, $\omega_{M E M}$ is memory evaluating parameter, $\omega_{D I S K}$ is disk evaluating parameter, $p_{* *}$ is the coefficient of the relative $* *$, and the $* *$ is load parameter derived by monitor.

Through the evolution of such parameter described above, updating administrator or updating management system will order the parameter according $\omega$ and select the light load nodes according a special scope to update firstly the new version service. The updating will be completed according to the similar rule until all the service in the system complete updating.

\subsection{States Management}

States management reorganizes a service's persistent state from the representation required by the old service to that required by the new service and the current service to that required by the past simulation service and future simulation service. Thus, client services do not notice that the service has upgraded, except that client services of the new type may see improved performance and fewer rejected requests, and client services of the old type may see decreased performance and more rejected requests. We adopted checkpointing technology [4][5][10][11] and process migration[12] technology to save the states of service states and recover the states for the new version service.

\subsection{Updating Transactions of Grid-Based System}

Due to the failure of updating procedure, the recovering of updating failure should be considered. The updating transaction is adopted for managing the updating failure. The updating mid-state is stored in the database through resources of updating information service.

When system updating failures, the administrator or updating managing system recover the system to the original point from the mid updating states stored in database and the updating of system seems doesn't have happen. So the system updating procedure is an atom transaction [9]. The checkpointing technology $[4][5][10][11]$ and process migration[12] technology are adopted as state saving technology and state recovering.

\section{Prototype and Analysis}

In order to validate the method's validity, we build a grid platform infrastructure which supports grid service dynamic updating. GT4[2] is adopted as the software platform and service is developed conform to WSRF[3] specification. The updating scheduling basing on monitor of computing resource with WS-MDS[2] will make the updating procedure more efficient through selecting more optimal updating subset of grid system nodes. The physical environment is showed as figure 5 . 


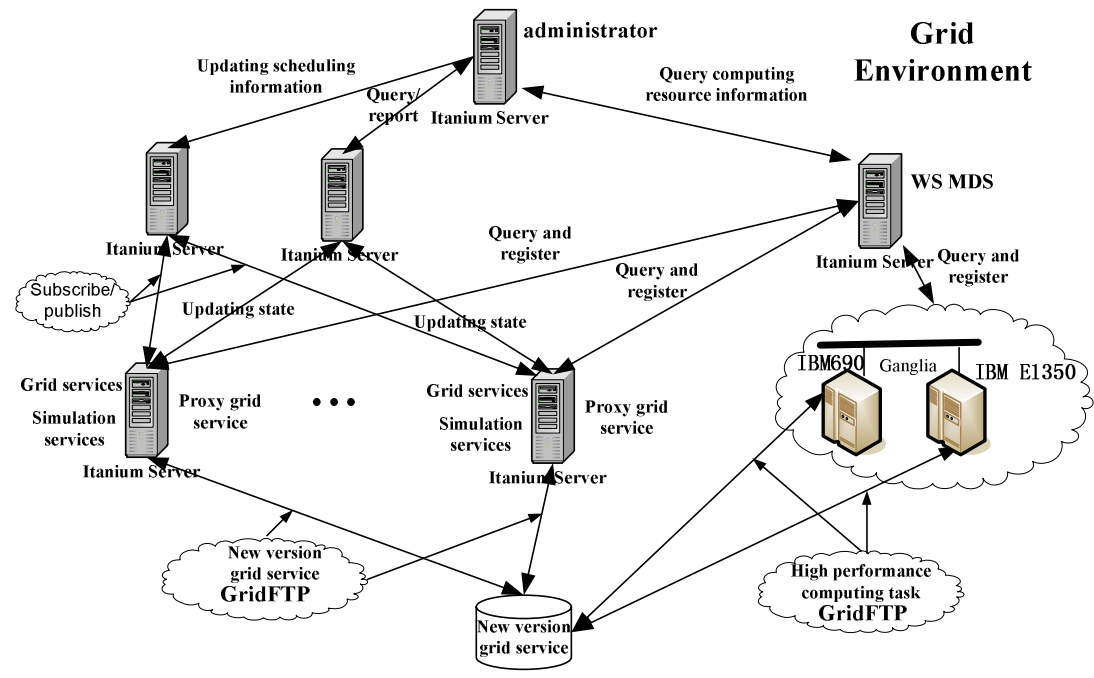

Fig. 5. Grid environment supporting service updating

\section{Summary and Future Work}

A grid service dynamic updating method in grid environment is presented in this paper and proxy service is introduced in this method for service request transmitting. The transparency between services could reach by introducing proxy service. The mechanism supporting multi version coexist at same time is introducing simulation service. Simulation service is responsible for simulating interface behavior and states format transferring of different versions.

In the aspect of state transferring, we adopt a mature state transfer method used in other updating system. In the future, we will research more suitable state transferring mechanism for system constructed by grid service.

Acknowledgments. This paper is supported by Grand 60673116 of National Natural Science Foundation of China, Grand 2006AA01Z166 of the National High Technology Research and Development Program of China (863).

\section{References}

1. Foster, I., Kesselman, C., Tuecke, S., "The Anatomy of the Grid: Enabling Scalable Virtual Organization", International Journal of Supercomputer Applications, 2001.3, Vol. 15(3), pp200-222

2. Globus Toolkit 4.0. http://www.globus.org/, 2006.11

3. WSRF-The WS-Resource Framework. http://www.globus.org/wsrf/, 2006.5

4. Michael Hicks. Dynamic Software Updating. PhD thesis, Computer and Information Science, University of Pennsylvania, 2001 
5. Peter Ebraert, Yves Vandewoude, Theo D'Hondt, Yolande Berbers. Pitfalls in unanticipated dynamic software evolution. Proceedings of the Workshop on Reflection, AOP and Meta-Data for Software Evolution(RAM-SE'05), 41-51

6. Yang Fu-qing, Mei Hong, Lu Jian, Jin Zhi. Some Discussion on the Development of Software Technology. Acta Electronica Sinica(in Chinese) ,2002, 30(12A):1901-1906

7. I Foster, C Kesselman. The Grid: Blueprint for a new computing infrastructure1.San Francisco: Morgan2Kaufmann ,1998

8. Ian Foster1, Carl Kesselman, et al. The Physiology of the Grid: An Open Grid Services Architecture for Distributed Systems Integration. http://www.globus.org/reserch/papers/ ogsa.pdf

9. Iulian Neamtiu, Michael Hicks, Gareth Stoyle, Manuel Oriol. Practical Dynamic Software Updating for C. Proceedings of the ACM Conference on Programming Language Design and Implementation (PLDI2006), pp72-83.

10. G. Bronevetsky, M. Schulz, P. Szwed, D. Marques, and K. Pingali. Application-level check pointing for shared memory programs. In Proc. ASPLOS, 2004.

11. J. S. Plank. An overview of checkpointing in uniprocessor and distributed systems, focusing on implementation and performance. Technical Report UT-CS-97-372, Computer Science Department, the University of Tennessee, 1997.

12. J. M. Smith. A survey of process migration mechanisms. ACM Operating Systems Review, SIGOPS, 22(3):28-40, 1988. 